\title{
EchoGéo
}

35 | 2016

Les découpages territoriaux en Europe

\section{Les découpages municipaux en Europe : la France est-elle vraiment une exception?}

Jean-Baptiste Grison

\section{OpenEdition}

1 Journals

Édition électronique

URL : https://journals.openedition.org/echogeo/14522

DOI : 10.4000/echogeo. 14522

ISSN : 1963-1197

Éditeur

Pôle de recherche pour l'organisation et la diffusion de l'information géographique (CNRS UMR 8586)

Référence électronique

Jean-Baptiste Grison, « Les découpages municipaux en Europe : la France est-elle vraiment une exception? », EchoGéo [En ligne], 35 | 2016, mis en ligne le 19 avril 2016, consulté le 11 août 2021

URL : http://journals.openedition.org/echogeo/14522 ; DOI : https://doi.org/10.4000/echogeo.14522

Ce document a été généré automatiquement le 11 août 2021.

EchoGéo est mis à disposition selon les termes de la licence Creative Commons Attribution - Pas d'Utilisation Commerciale - Pas de Modification 4.0 International (CC BY-NC-ND) 


\title{
Les découpages municipaux en Europe : la France est-elle vraiment une exception?
}

\author{
Jean-Baptiste Grison
}

1 Avec ses quelque 36000 communes, la France est régulièrement considérée comme le pays de l'émiettement territorial par excellence, au sein de l'Union Européenne, voire de l'Europe dans son ensemble. Cette exception française est reprise régulièrement dans les publications (Ziller, 2000 ; Guigou, 2000 ; Aubelle, 2011...), mettant en avant, par pays, les moyennes globales de population et superficie des unités administratives locales (LAU2). Il est vrai que les chiffres nationaux placent la France bien au-delà des tendances continentales, en matière de morcellement administratif.

2 Cependant, le reste de l'Europe est loin de présenter une organisation homogène, et chaque pays présente des spécificités propres. La qualité d'exception souvent attribuée à la France doit être confrontée aux multiples contextes nationaux : finalement, chaque trame administrative ne peut-elle pas, pour diverses raisons, être considérée comme exceptionnelle? Les comparaisons doivent être menées à plusieurs niveaux: d'une part, les moyennes générales du nombre, de la population et de la superficie des municipalités dans les différents pays; d'autre part, la variabilité interne de ces critères, à commencer par la répartition des communes par classes de population ; enfin, le statut et les compétences des entités de base sont à prendre en considération, dans la mesure où celles-ci n'ont pas la même importance d'un État à l'autre.

3 L'objectif de cet article est de caractériser et nuancer l'exception française, à partir de la diversité des maillages européens. Nous nous appuyons pour cela sur les moyennes par pays fournies par Eurostat, mais aussi sur la diversité morphologique et la variabilité démographique internes à chaque État, qui permettent d'affiner considérablement les diagnostics. Nous faisons ici le choix de centrer nos propos sur la strate municipale, niveau de base de l'administration locale, sans perdre de vue l'idée que la comparaison des systèmes administratifs territoriaux dans leur ensemble, toutes 
échelles confondues, peut aussi poser la question de l'exception française (Giraut, 2002).

\section{La comparaison des moyennes nationales et l'évolution des maillages : une convergence variable selon les critères employés}

4 À travers l'Europe, la trame municipale est caractérisée par une instabilité plus ou moins marquée. Aucun État européen ne peut revendiquer un maillage local immuable, tous connaissent des aménagements chroniques, qu'ils soient marginaux ou généralisés. Par ailleurs, la tendance dominante depuis plus d'un siècle est à la diminution, d'ampleur variable suivant les pays, du nombre de collectivités locales.

5 Les successions de réformes, si elles s'observent depuis le début du dix-neuvième siècle, ont eu tendance à s'accélérer au cours de la seconde moitié du vingtième siècle. Inégales d'un pays à l'autre, voire d'une région à l'autre au sein d'un même pays, elles ont pour conséquence une hétérogénéité croissante de la trame locale européenne, qui rend les comparaisons de plus en plus difficiles.

6 En conséquence de ces évolutions, la trame des localités, correspondant au réseau historique des communautés locales (souvent paroissiales), est de moins en moins lisible. Avant même d'aborder la variabilité des compétences et statuts de l'unité administrative locale, comment comparer une commune rurale française, composée d'un seul village, à une commune suédoise rassemblant plusieurs dizaines d'anciennes paroisses?

7 L'office européen des statistiques Eurostat a mis au point, dans cette optique de comparaison, une grille de lecture des unités statistiques à cinq niveaux, dénommés NUTS 1, NUTS 2 et NUTS 3 pour les unités de dimension régionale et sous régionale, LAU 1 et LAU 2 pour les unités administratives locales, le dernier étant le niveau le plus fin. La France est bien entendu celui qui en compte le plus (36 556 communes en 2011)1.

8 Pour chaque pays considéré (ceux de l'Union Européenne, ainsi que l'Islande, le Liechtenstein, la Norvège et la Suisse, soit le panel pris en compte par l'office Eurostat) nous avons collecté le nombre d'unités administratives relevant du niveau statistique LAU 2 (parfois dénommé NUTS5). Le tableau 1 rend compte des populations et superficies moyennes de ces unités pour chacun de ces pays. Au total, 121412 communes ou équivalents sont recensées, ce qui signifie que la France pèse pour un peu moins d'un tiers du total $(30,1 \%)$, mais avec seulement $13 \%$ de la population et un peu moins de la superficie. Dans l'ensemble, il est donc évident que le morcellement municipal y est sensiblement plus important que la moyenne.

9 Cependant, une première difficulté de comparaison apparait dans le fait que plusieurs pays d'Europe ont adopté deux niveaux de proximité, le niveau municipal n'étant pas le plus fin. Ainsi, au Royaume-Uni, en Irlande, au Danemark, au Portugal, en Bulgarie, en Grèce, en Slovénie, en Lituanie, les collectivités municipales sont enregistrées au niveau LAU 1, alors qu'un niveau inférieur continue d'exister avec un statut réduit. Lequel des deux doit être considéré comme l'équivalent de la commune française et employé dans les comparaisons? Les études positionnant la France en Europe varient de manière significative suivant l'option retenue: le nombre de municipalités européennes peut passer, en employant les critères de statut les plus restrictifs, en-dessous de 90000. 
10 La population moyenne par entité de base se situe, en France, parmi les plus basses des pays européens. On peut ainsi ranger l'Hexagone au sein d'un groupe inférieur comprenant six autres États à moins de 2500 habitants par entité : le Portugal, la Slovaquie, la Grèce, la République Tchèque, Chypre et l'Irlande. Du côté de la superficie moyenne des mailles locales, les chiffres français sont proches de ceux de la République Tchèque, de la Slovaquie ou de la Suisse (entre 12 et $17 \mathrm{~km}^{2}$ ); Malte présente une moyenne très basse $(4,71)$ reflétant sa forte densité de population, tandis qu'un groupe de cinq pays offre des chiffres légèrement supérieurs mais comparables (entre 20 et $\left.26 \mathrm{~km}^{2}\right)$ : il s'agit de l'Irlande, de Chypre, de la Grèce, du Portugal, du Luxembourg, ainsi que du Royaume-Uni, du Danemark et de la Bulgarie.

11 La population médiane des unités administratives locales respecte dans ses grandes lignes la hiérarchie avancée par les moyennes démographiques. Une exception notable est cependant à relever, celle de l'Espagne dont la commune médiane se rapproche des pays aux chiffres les plus faibles, n'étant que légèrement supérieure au cas français, lequel est encore voisin de la République Tchèque et de la Grèce. Chypre et la Bulgarie font exception avec une médiane très faible.

12 Les écarts-types de la population des unités locales apportent en outre une information intéressante. Les valeurs les plus faibles correspondent, d'une part, à Malte et au Liechtenstein, petits pays à la trame relativement homogène et, d'autre part, au Royaume-Uni, à l'Irlande, au Danemark et au Portugal. En effet, dans ces quatre pays, le niveau «LAU 2 » n'est pas une collectivité territoriale à part entière, mais joue le rôle de circonscription électorale: il subdivise donc la plupart des villes. Chypre et la Slovaquie ont un écart-type faible lié à la rareté des grandes villes. En situation intermédiaire (écart-type entre 10000 et 20000 ), se trouvent des trames locales assez comparables à celle de la France, plutôt morcelées en milieu rural, mais comportant aussi des grandes villes administrativement unifiées. Les écarts-types les plus importants s'observent enfin, presque tous, dans des trames locales largement réformées (peu de morcellement rural), avec deux exceptions notoires: l'Espagne et l'Italie, aux maillages stables mais caractérisés par une très forte hétérogénéité villecampagne et interrégionale.

13 Au premier abord et au regard de ces chiffres nationaux, il convient donc de relativiser le caractère exceptionnel de la situation française, même s'il est vrai que seule une minorité de pays se situe dans les moyennes les plus faibles pour les deux indicateurs (étendue des mailles et population). Nous devons surtout retenir la grande diversité des maillages et l'absence de "modèle unique » qui pourrait s'imposer en dehors du cas français.

Tableau 1 - Les unités administratives de base dans les pays d'Europe : quelques éléments de comparaison

\begin{tabular}{|l|l|l|l|l|l|l|l|}
\hline \multirow{2}{*}{ pays } & Population & Superficie & $\begin{array}{l}\text { nombre } \\
\text { d'unités } \\
\text { LAU2 }\end{array}$ & $\begin{array}{l}\text { population } \\
\text { moyenne }\end{array}$ & $\begin{array}{l}\text { luperficie } \\
\text { moyenne } \\
\left(\mathrm{km}^{2}\right)\end{array}$ & $\begin{array}{l}\text { Population } \\
\text { médiane }\end{array}$ & $\begin{array}{l}\text { Ecart- } \\
\text { type }\end{array}$ \\
\cline { 2 - 8 } & $2011)$ & $\left(\mathrm{km}^{2}\right)$ & & & & & \\
\hline Allemagne & 80219695 & 357020 & 11329 & 7081 & 31,5 & 1664 & 45988 \\
\hline
\end{tabular}




\begin{tabular}{|c|c|c|c|c|c|c|c|}
\hline Autriche & 8401940 & 83870 & 2357 & 3565 & 35,6 & 1571 & 36880 \\
\hline Belgique & 11000638 & 30530 & 589 & 18677 & 51,8 & 12009 & 30792 \\
\hline Bulgarie* & 7364570 & 110910 & 4619 & 1594 & 24,0 & 213 & 18892 \\
\hline Chypre* & 840407 & 9250 & 402 & 2091 & 23,0 & 208 & 6848 \\
\hline Croatie & 4284889 & 56540 & 556 & 7707 & 101,7 & 2769 & 35385 \\
\hline Danemark* & 5560628 & 43090 & 2116 & 2628 & 20,4 & 934 & 3311 \\
\hline Espagne & 46815910 & 505580 & 8111 & 5772 & 62,3 & 564 & 46907 \\
\hline Estonie & 1294455 & 45230 & 226 & 5728 & 200,1 & 1575 & 27363 \\
\hline Finlande & 5375276 & 338150 & 336 & 15998 & 1006,4 & 5849 & 44653 \\
\hline France & 64933400 & 551500 & 36556 & 1776 & 15,1 & 430 & 14759 \\
\hline Grèce* & 10816286 & 131940 & 6136 & 1763 & 21,5 & 346 & 12049 \\
\hline Hongrie & 9937628 & 93030 & 3176 & 3129 & 29,3 & 837 & 11262 \\
\hline Irlande* & 4574888 & 70280 & 3405 & 1344 & 20,6 & 613 & 2255 \\
\hline Islande & 315556 & 103000 & 75 & 4207 & 1373,3 & 890 & 14333 \\
\hline Italie & 59433744 & 301230 & 8092 & 7345 & 37,2 & 2437 & 39739 \\
\hline Lettonie & 2070371 & 64590 & 119 & 17398 & 542,8 & 6780 & 59257 \\
\hline Liechtenstein & 36149 & 160 & 11 & 3286 & 14,5 & 3999 & 1744 \\
\hline Lituanie* & 3043429 & 65200 & 560 & 5435 & 116,4 & 2236 & 11689 \\
\hline Luxembourg & 512353 & 2590 & 106 & 4834 & 24,4 & 2418 & 10648 \\
\hline Malte & 417432 & 320 & 68 & 6139 & 4,7 & 3973 & 4905 \\
\hline Norvège & 4979956 & 324220 & 429 & 11608 & 755,8 & 4570 & 34742 \\
\hline Pays-Bas & 16655799 & 41530 & 418 & 39846 & 99,4 & 25599 & 64168 \\
\hline Pologne & 38044565 & 312690 & 2479 & 15347 & 126,1 & 7533 & 50455 \\
\hline Portugal* & 10562178 & 92120 & 4260 & 2479 & 21,6 & 892 & 5086 \\
\hline $\begin{array}{l}\text { République } \\
\text { Tchèque }\end{array}$ & 10436560 & 78870 & 6251 & 1670 & 12,6 & 424 & 17689 \\
\hline Roumanie & 20121641 & 238390 & 3181 & 6326 & 74,9 & 3118 & 38704 \\
\hline
\end{tabular}




\begin{tabular}{|l|l|l|l|l|l|l|l|}
\hline $\begin{array}{l}\text { Royaume- } \\
\text { Uni* }\end{array}$ & 63182180 & 243610 & 9523 & 6635 & 25,6 & 4979 & 1614 \\
\hline Slovaquie & 5399333 & 49030 & 2927 & 1845 & 16,8 & 654 & 5843 \\
\hline Slovénie & 2050189 & 20270 & 210 & 9763 & 96,5 & 4786 & 21937 \\
\hline Suède & 9482855 & 450290 & 290 & 32700 & 1552,7 & 15283 & 44722 \\
\hline Suisse & 6587556 & 41290 & 2499 & 2636 & 16,5 & 1224 & 11009 \\
\hline
\end{tabular}

*=Le niveau LAU 2 est un niveau infra-municipal.

Données : EUROSTAT 2011

\section{Morphologie des maillages communaux : des situations contrastées}

14 Au-delà des moyennes globales, les formes et la nature des maillages municipaux sont des éléments importants de différenciation. Il s'agit d'aborder, d'une part, la nature de la relation entre la trame administrative et la répartition de l'habitat, qui renvoie aux conditions de sa formation : la commune correspond-elle aux anciennes paroisses, aux unités d'habitat de base (villages et bourgs), ou rassemble-t-elle plusieurs localités? Dans cette optique, plusieurs cas de figure peuvent être rencontrés, en considérant : la répartition traditionnelle de l'habitat (degré de dispersion de la population); l'ancienneté du maillage actuel (hérité ou réformé); le degré d'homogénéité et l'ampleur des différenciations régionales internes; la diversité des statuts de la collectivité de base.

\section{Le poids des formes de répartition de l'habitat}

15 Dans la plupart des pays, comme d'ailleurs en France, la trame des cellules administratives de base s'est constituée, initialement, autour des noyaux de concentration de l'habitat qui reprennent, le plus souvent, la trame des anciennes paroisses. L'hétérogénéité interne au maillage communal français a déjà été explorée sous cet angle (Meynier, 1945; Delamarre, 1989). Tous les grands pays européens connaissent des nuances régionales plus ou moins marquées de ce point de vue. Plusieurs types de maillages s'opposent ainsi.

- Le maillage très morcelé des régions où l'habitat est groupé en villages de tailles modestes : cas typique du nord-est de la France, de la Rhénanie-Palatinat en Allemagne, par exemple.

- Les maillages composés de fermes isolées organisées autour des petites paroisses rurales, comme cela peut être observé, en France, en Normandie ou Gascogne, ou dans de nombreuses vallées alpines (des Préalpes françaises au Tyrol autrichien) et les îles Britanniques.

- Le maillage plus lâche de régions de tradition urbaine forte, où l'habitat est très centralisé dans des bourgs ou petites villes (incluant ou non une dispersion périphérique en hameaux ou fermes isolées): c'est le cas de nombreuses régions méditerranéennes (Andalousie, 
Murcie, basse Provence, une grande partie de l'Italie), mais aussi des Pays-Bas et de certaines régions allemandes.

- Le maillage, lâche également, de régions d'habitat dispersé en hameaux et fermes isolées, au sein desquels la fréquence de bourgs-centres est plus rare. En France, il s'agit plus particulièrement de la Bretagne et de la majeure partie du Massif central.

16 L'histoire de la mise en place des trames municipales varie selon les pays, de telle manière que ces aspects d'organisation de l'habitat ont des influences variables sur les maillages actuels. Dans l'ensemble, le rôle de la morphologie des zones habitées s'est considérablement amenuisé dans les États ayant mené des processus de fusions communales de grande ampleur.

\section{L'ancienneté du maillage et l'impact des remodelages contemporains}

17 Les trames nationales actuelles de l'administration locale proviennent d'héritages variables. En particulier, une partie des pays européens a choisi de conduire une réforme radicale de ses institutions locales, conduisant à une réduction sensible du nombre de municipalités. L'exemple voisin le plus clair est celui de la Belgique qui, lors d'une réforme en 1974, divise par cinq le nombre d'entités de base en agrégeant, en milieu rural, les villages périphériques aux bourgs et petites villes qui les polarisent. Plusieurs autres pays ont suivi cette direction, les réductions les plus importantes s'observant au Danemark, en Suède, mais aussi en Bulgarie et en Allemagne, bien que, dans ce dernier cas, la réduction ait été plus progressive et inégalement répartie. La Grèce a procédé à des réformes importantes au début du XXI ${ }^{\mathrm{e}}$ siècle, après avoir longtemps fait reposer les institutions municipales sur une trame très comparable à celle de la France. Ces anciennes municipalités, qui sont toujours enregistrées par Eurostat au niveau LAU 2, ont désormais le titre de "communautés locales » dotées d'un conseil au rôle essentiellement consultatif.

Dans d'autres États, des fusions ont eu lieu de manière plus modérée, si bien que l'aspect général de leur trame n'est pas réellement bouleversé. Il en est ainsi pour les Pays-Bas, certaines régions d'Autriche, la Pologne, la Roumanie. En revanche, l'essentiel des trames des pays méditerranéens (Grèce mise à part) a connu une relative stabilité, le nombre de municipalités ayant même légèrement augmenté en Italie.

19 Dans les pays de l'Europe centrale et orientale, qui ont rejoint récemment l'Union Européenne, les trames sont généralement très instables. Le système communiste y avait mis en place des organisations que les gouvernements postérieurs à l'effondrement du « rideau de fer " n'ont pas toujours souhaité conserver. Quand ils l'ont été (République Tchèque, Hongrie, Slovaquie), on assiste chaque année à une série de créations ou fusions d'entités (Maurel, 2005 ; Depraz, 2005).

\section{L'importance des différenciations régionales}

20 En plus d'être très différents d'un pays à l'autre, les maillages administratifs locaux sont parfois très hétérogènes au sein d'un même État. Les régimes fédéraux montrent les exemples les plus flagrants, à commencer par l'Allemagne, dont la baisse sensible, globalement, du nombre de municipalités, cache des réalités très différentes d'un Land à l'autre. Ainsi, dans la région frontalière de Rhénanie-Palatinat, aucune refonte de la 
trame des communes n'a été engagée, si bien que leur nombre est resté élevé, autour de 2300 (pour quatre millions d'habitants), ce qui signifie un morcellement considérable, proche de la situation de l'Alsace voisine. En revanche, les Länder de Sarre, Hesse ou Rhénanie-du-Nord-Westphalie ont connu des mouvements très importants de fusions municipales, comparables à ce qui a été fait en Belgique. L'État le plus peuplé d'Allemagne ne compte plus que 373 communes pour 17 millions d'habitants. En situation intermédiaire, le Bade-Wurtemberg et la Bavière ont privilégié des regroupements plus légers, par deux ou trois entités. Avec quelques décennies de retard, les nouveaux Länder de l'Allemagne de l'Est reproduisent cette diversité de situations. Avec une évolution générale moins marquée, l'Autriche aboutit à un résultat similaire : la Styrie et le Tyrol conservent de nombreuses petites communes, tandis que la Carinthie et le Vorarlberg ont procédé à des réformes importantes.

21 L'Espagne et l'Italie connaissent aussi des divergences régionales marquées de leur trame municipale, malgré l'absence de réformes au cours des dernières décennies. Correspondant globalement à un gradient nord-sud, elles sont liées, avant tout, à des variations dans l'organisation traditionnelle des sociétés locales et des structures de l'habitat qui y sont liées. En Espagne, on peut relever par exemple le contraste entre Murcie (45 communes pour $11317 \mathrm{~km}^{2}$ et 1 million d'habitants) et La Rioja (174 communes pour $5034 \mathrm{~km}^{2}$ et 263000 habitants). Ce contraste ne s'explique pas par une politique de regroupement plus poussée dans l'une des deux régions, ni par des conceptions divergentes de l'entité municipale. Tout simplement, la répartition de l'habitat traditionnel est différente, de telle sorte que la population de Murcie est largement centralisée sur des villes et gros bourgs, tandis qu'on observe dans la Rioja une multiplicité de petits villages qui sont autant d'anciennes paroisses.

\section{Les statuts des unités administratives locales : une grande hétérogénéité}

22 Les niveaux de décision de la politique locale sont multiples dans presque tous les pays du continent européen. Les nomenclatures d'EUROSTAT renseignent d'ailleurs le plus souvent les deux niveaux (LAU 1 et LAU 2), le premier correspondant majoritairement à des regroupements de type intercommunal. Seuls certains territoires ayant des trames récemment réformées n'ont qu'une seule strate municipale (Belgique, Pays-Bas, Slovénie, Suède...). Dans les autres États, le partage des compétences entre les deux échelons locaux est un facteur clé de différenciation. En France, le niveau LAU 1 est resté adossé à celui des cantons, bien que les intercommunalités prennent aujourd'hui une place plus importante dans l'organisation locale des territoires.

23 Une partie des pays a choisi de transférer le niveau municipal vers un échelon plus large que la trame de base des localités. C'est ainsi que, au Danemark, en Bulgarie, en Irlande et au Royaume-Uni, le nombre de municipalités a considérablement diminué, par création d'une couche supplémentaire, le maillage de base conservant un statut particulier, même si l'essentiel des fonctions politiques et administratives ne lui appartiennent plus. Au Portugal, un tel système existe depuis déjà longtemps (freguesia et concelho). Dans les îles Britanniques, le Local government act instituant ce transfert de statut remonte à la fin du dix-neuvième siècle. Dans ces deux États, on peut ainsi affirmer que la municipalité, telle qu'elle est envisagée ailleurs, n'existe pas. Les districts 
n'en ont pas la forme, les wards et civil parishes («paroisses civiles ») n'en ont pas le statut.

Dans les États fédéraux, en particulier en Allemagne, plusieurs systèmes se côtoient selon les Länder, si bien qu'il est difficile de parler d'une position dominante. Il apparaît par exemple que l'intercommunalité est assez largement développée dans des régions où la trame communale est restée morcelée (Verbandsgemeinde en Rhénanie-Palatinat, Amt en Schleswig-Holstein, Verwaltungsgemeinschaft en Thüringe...), alors qu'elle est inexistante, en-deçà de l'échelle de l'arrondissement (Kreis) dans les Länder où le maillage communal a fait l'objet de fusions massives (Hesse, Sarre, Rhénanie-du-NordWesstphalie). La situation autrichienne est assez proche, avec cependant des divergences un peu moins prononcées. En Espagne, la répartition des pouvoirs entre les deux niveaux locaux varie aussi suivant les communautés autonomes et le poids spécifique attribué aux Comarcas.

En somme, si la plupart des pays d'Europe possède bien deux niveaux d'action locale, il y a une certaine opposition entre les États qui articulent communes et intercommunalités, et ceux pour lesquels ce sont les communes, plus vastes, qui sont subdivisées en organisations infracommunales. Seuls quelques pays, qui ne sont pas parmi les plus peuplés, n'ont pas retenu ce double niveau local : la Belgique, la Suède, la Slovénie... Cela engendre un inévitable déséquilibre dans les comparaisons interétatiques, qui imposent de choisir entre une équivalence de statut et une équivalence de maille.

\section{Les très petites communes en Europe}

26 Les moyennes globales de population des unités administratives locales ne permettent pas de mesurer la diversité de la taille des entités. Si l'on considère que des problèmes de gouvernance sont à même de survenir dans des municipalités très peu peuplées, il convient d'approfondir cet aspect. Quels pays européens sont confrontés aux très petites entités? Dans quelle mesure le cas français est-il aussi exceptionnel sur ce point? Existe-t-il des comparaisons possibles avec d'autres situations européennes? Une approche à partir des communes de moins de cent et moins de cinquante habitants permet d'apporter quelques éléments de réponse. Nous avons retenu, volontairement, ces seuils démographiques très bas, dans la mesure où ils traduisent, à notre sens, un degré avancé de morcellement municipal, surtout lorsque les entités concernées sont concentrées dans des régions bien définies. Par ailleurs, des seuils très bas permettent de se focaliser sur des communes dont le statut renvoie bien, dans tous les territoires, à celui d'entités périphériques: dans certains territoires ruraux, les bourgs-centres peuvent compter moins de 500 habitants (Grison, 2012).

Dans l'ensemble, il apparaît que, en valeur absolue, le nombre de communes de moins de cent ou moins de cinquante habitants en France est inégalé ailleurs. Notre pays compte en effet $36 \%$ des entités de moins de cinquante habitants et $4 \%$ de celles de moins de cent, pour $30 \%$ des unités administratives locales de la zone étudiée. Cependant, des zones de concentration de petites entités existent aussi dans d'autres pays, et il est important de les évoquer. 
Tableau 2 - Les très petites unités administratives dans les pays de l'Union européenne

\begin{tabular}{|c|c|c|c|c|c|}
\hline Pays & $\begin{array}{l}\text { Nombre } \\
\text { d'unités } \\
\text { LAU } 2\end{array}$ & $\begin{array}{l}\text { Unités de moins } \\
\text { de } 100 \text { habitants }\end{array}$ & $\begin{array}{lr}\text { Unités } & \text { de } \\
\text { moins } & \text { de } \\
50 \text { habitants } & \end{array}$ & $\begin{array}{lr}\text { Part des } & \text { de } \\
\text { unités } & \text { de } \\
\text { moins de } & 100 \\
\text { habitants }\end{array}$ & $\begin{array}{l}\text { Part des unités } \\
\text { de moins de } \\
50 \text { habitants }\end{array}$ \\
\hline Allemagne & 11329 & 209 & 53 & $1,8 \%$ & $0,5 \%$ \\
\hline Autriche & 2357 & 6 & 0 & $0,3 \%$ & $0,0 \%$ \\
\hline Belgique & 589 & 1 & 0 & $0,2 \%$ & $0,0 \%$ \\
\hline Bulgarie* & 4619 & 1046 & 544 & $22,6 \%$ & $11,8 \%$ \\
\hline Chypre* & 614 & 100 & 53 & $16,3 \%$ & $8,6 \%$ \\
\hline Croatie & 556 & 0 & 0 & $0,0 \%$ & $0,0 \%$ \\
\hline Danemark $^{*}$ & 2116 & 22 & 6 & $1,0 \%$ & $0,3 \%$ \\
\hline Espagne & 8116 & 1104 & 411 & $13,6 \%$ & $5,1 \%$ \\
\hline Estonie & 226 & 2 & 0 & $0,9 \%$ & $0,0 \%$ \\
\hline Finlande & 336 & 0 & 0 & $0,0 \%$ & $0,0 \%$ \\
\hline France & 36685 & 3487 & 893 & $9,5 \%$ & $2,4 \%$ \\
\hline Grèce* & 6136 & 934 & 335 & $15,2 \%$ & $5,5 \%$ \\
\hline Hongrie & 3176 & 151 & 43 & $4,8 \%$ & $1,4 \%$ \\
\hline Irlande $^{*}$ & 3405 & 30 & 0 & $0,9 \%$ & $0,0 \%$ \\
\hline Islande & 75 & 5 & 0 & $6,7 \%$ & $0,0 \%$ \\
\hline Italie & 8092 & 53 & 6 & $0,7 \%$ & $0,1 \%$ \\
\hline Lettonie & 119 & 0 & 0 & $0,0 \%$ & $0,0 \%$ \\
\hline Lituanie $^{*}$ & 540 & 0 & 0 & $0,0 \%$ & $0,0 \%$ \\
\hline Liechtenstein & 11 & 0 & 0 & $0,0 \%$ & $0,0 \%$ \\
\hline Luxembourg & 106 & 0 & 0 & $0,0 \%$ & $0,0 \%$ \\
\hline Malte & 68 & 0 & 0 & $0,0 \%$ & $0,0 \%$ \\
\hline Norvège & 429 & 0 & 0 & $0,0 \%$ & $0,0 \%$ \\
\hline Pays-Bas & 418 & 0 & 0 & $0,0 \%$ & $0,0 \%$ \\
\hline Pologne & 2479 & 0 & 0 & $0,0 \%$ & $0,0 \%$ \\
\hline
\end{tabular}




\begin{tabular}{|c|c|c|c|c|c|}
\hline Portugal $^{*}$ & 4260 & 62 & 6 & $1,5 \%$ & $0,1 \%$ \\
\hline $\begin{array}{l}\text { République } \\
\text { Tchèque }\end{array}$ & 6251 & 464 & 66 & $7,4 \%$ & $1,1 \%$ \\
\hline Roumanie & 3131 & 1 & 0 & $0,0 \%$ & $0,0 \%$ \\
\hline Royaume-Uni ${ }^{*}$ & 9523 & 0 & 0 & $0,0 \%$ & $0,0 \%$ \\
\hline Slovaquie & 2927 & 123 & 31 & $4,2 \%$ & $1,1 \%$ \\
\hline Slovénie & 211 & 0 & 0 & $0,0 \%$ & $0,0 \%$ \\
\hline Suède & 290 & 0 & 0 & $0,0 \%$ & $0,0 \%$ \\
\hline Suisse & 2499 & 71 & 25 & $2,8 \%$ & $1,0 \%$ \\
\hline
\end{tabular}

*=Le niveau LAU 2 est un niveau infra municipal.

Source : EUROSTAT 2011.

28 Au total, une douzaine de pays sur les trente-deux étudiés comptent des communes (ou juridiction équivalente) de moins de cinquante habitants (tableau 2). Si on élève le seuil à la centaine de résidents, on aboutit à une vingtaine d'États concernés, soit un peu plus de la moitié. Sur cet ensemble, la majorité n'a en fait qu'une poignée d'entités, qui correspondent par conséquent à des situations d'exception : la Belgique compte ainsi une municipalité de 85 habitants en 2000, dans la province du Limbourg, ayant échappé à la réforme des années 1970 pour cause de spécificité linguistique. L'Estonie n'en a que deux, sur sept de moins de 500 habitants, ce qui signifie aussi qu'il s'agit de cas très particuliers. De même, il en existe une en Roumanie. On en trouve encore cinq en Autriche, dans le cadre d'une répartition globale davantage tirée vers le bas (172 municipalités de moins de 500 résidents).

29 Les nations davantage concernées par le phénomène sont l'Allemagne, Chypre, l'Irlande, l'Espagne, la France et le Portugal. Il faut ajouter les cas de la Grèce et de la Bulgarie, qui comptent des proportions considérables de très petites entités, mais ces dernières n'ont conservé que très peu de pouvoirs (rôle principalement représentatif) depuis les dernières réformes (Boulineau, 2003 ; Sivignon et al. 2003). L'Italie est dans une situation intermédiaire, avec une trentaine de communes de moins de cent habitants (dont six de moins de cinquante). Les trois exemples que nous développons correspondent à trois États ayant des très petites communes dans des maillages de types différents: trame ancienne pour l'Espagne, en grande partie réformée dans les dernières décennies pour l'Allemagne, à double niveau pour le Portugal.

\section{L'Espagne : des municipalités entre grandeur et misère}

30 L'Espagne comptait 8116 municipalités au recensement de 2011 (contre 8077 en 2001), dont 1104 (13,6\%) de moins de 100 habitants, et 411 (5,1\%) de moins de 50. Les deux valeurs relatives sont supérieures aux valeurs françaises correspondantes (respectivement 9,5 et 2,4\%). Par ailleurs, la répartition de ces très petites communes 
espagnoles par province (illustration 1) montre de profondes divergences et fait apparaître des zones de concentrations majeures, avec des inégalités de répartition sensiblement plus prononcées qu'en France. En outre, et contrairement à la France, le nombre et la part de très petites communes ont sensiblement augmenté au cours des années 2000.

Illustration 1- Les communes de moins de cinquante habitants en Espagne



31 Les comparaisons hâtives des trames administratives française et espagnole sont loin de provoquer un tel constat: l'Espagne compte près de 5 fois moins de municipalités que la France, pour une superficie inférieure seulement de $10 \%$. La surface moyenne de ses communes y est sensiblement plus élevée $\left(62 \mathrm{~km}^{2}\right.$ contre 15 pour la France), de même que la population moyenne (5 770 habitants contre 1780$)^{2}$. Cependant, une analyse un peu plus approfondie montre que les inégalités dans la répartition de la population sont particulièrement marquées, avec, dans le centre du pays, des plateaux où les densités de population ont chuté à des niveaux très bas. A l'opposé de la hiérarchie des lieux, les municipalités espagnoles de plus de 100000 habitants, au nombre de 54 en 2001, concentrent à elles seules près de $40 \%$ de la population du pays (moins de $15 \%$ en France).

Après la restauration de la démocratie en 1975, les collectivités territoriales sont reparties sur des bases nouvelles, le flou des premières années se résorbant progressivement par un enrichissement de la législation et une autodétermination graduelle des différentes structures territoriales (Gabriel-Oyhamburu, 2000; Sanz Gomez, 2005). Cependant, des problèmes demeurent quant au traitement des distorsions entre les grandes villes à très fort pouvoir budgétaire (qui réclament des compétences supplémentaires) et les petites communes qui peinent à assurer leurs compétences minimales. En fait, le débat semble toujours d'actualité, d'autant plus que 
les fusions de communes, si elles n'ont pas été inexistantes depuis le rétablissement de la monarchie, sont restées relativement marginales, un peu à l'instar du cas français.

La situation espagnole est sans doute celle qui, en Europe, présente le plus de similitude avec le cas français en matière de très petites collectivités - du moins dans le centre-nord du pays. Même si les régions concernées sont davantage limitées dans l'espace, leur concentration et leur statut rapprochent les deux problématiques nationales. Les tendances contemporaines sont peut-être même plus préoccupantes dans la péninsule Ibérique, où les écarts se creusent toujours entre les grandes villes et les plateaux très dépeuplés, sur lesquels les densités de population sont tombées à des niveaux très bas, ne connaissent pas encore le renouveau qui s'observe dans la majorité des espaces ruraux français, même si, ponctuellement, des reconquêtes de villages par des populations d'origine étrangères sont observées (Nates Cruz, 2008).

\section{L'Allemagne : le poids déterminant des contextes régionaux}

\section{Une organisation et une modernité administratives souvent citées en exemple...}

35 L'organisation administrative de l'Allemagne est souvent citée en exemple (Guigou, 2000), comme une trame rigoureuse, régulière et efficace en termes d'aménagement et de développement $\mathrm{du}$ territoire. Les régions sont puissantes, les arrondissements (Kreise) correspondent bien aux bassins de vie, et les communes sont peuplées (plus de 6000 habitants en moyenne nationale, soit près de quatre fois le niveau français), leur nombre ayant diminué de moitié depuis les années 1970. Contrairement à la France, le statut des unités municipales n'est pas homogène, mais se partage en deux grands types : les villes (Städte) se distinguent des autres communes (Gemeinden). Les premières ont des compétences élargies, ce qui constitue une forme de réponse au problème, rencontré en Espagne ou en France, de disparités démographiques et financières entre municipalités. Certaines d'entre elles (112 en 2003) sont en outre des villesarrondissements (Kreisfreie Städte), ce qui leur permet de cumuler les compétences de deux niveaux administratifs.

\section{...Mais des irrégularités profondes, y compris au sein de l'ancienne Allemagne} fédérale

Malgré les impressions de premier abord, à y regarder de près, la République fédérale n'est pas tout à fait en reste en matière de très petites communes. Le cas le plus intéressant est celui du Land de Rhénanie-Palatinat, qui compte, en 2003, 126 municipalités de moins de cent habitants sur 2305 (5,5\%), dont 39 de moins de 50 habitants, mais surtout où 72 de ces entités sont concentrées dans le même arrondissement (le Kreis de Bitburg-Prüm, frontalier avec le Luxembourg et la Belgique) où elles représentent $30,6 \%$ des communes, et dont trente collectivités ont moins de cinquante habitants $(12,8 \%)^{3}$. Deux districts, le long de la vallée de l'Our qui marque la frontière germano-luxembourgeoise, ont une majorité de communes de moins de cent habitants. Ce secteur représente depuis déjà longtemps une véritable originalité en Allemagne (Reitel, 1973).

Sans que l'émiettement de la trame de base n'entrâne une prolifération aussi significative des structures de moins de 100 habitants, les municipalités sont restées au niveau des paroisses, sans réforme de la trame de base de l'administration, dans deux autres Länder : le Schleswig-Holstein et la Thuringe. Dans l'ensemble de l'État fédéral, environ 200 entités se trouvent en dessous du seuil de la centaine de résidents 
(illustration 2). Cependant, les régions concernées ont toutes conservé (ou mis en place) des regroupements intermédiaires entre l'arrondissement et la commune, qui peuvent centraliser l'essentiel des pouvoirs politiques locaux. Par ailleurs, les nouveaux Länder issus de l'ex-RDA conduisent encore, à la fin des années 2000, des réformes réduisant le nombre de très petites collectivités (Depraz, 2006).

La concentration de très petites communes à la frontière luxembourgeoise, même si elle reste ponctuelle, est comparable aux fréquences importantes de certains départements français (Grison, 2012), et présente un intérêt singulier, dans la mesure où l'administration allemande est fondée sur un modèle fédéral très différent du modèle français, et où les niveaux principaux de décision sont situés à des échelles différentes (Länder, Kreise, communes) des niveaux principaux français (État, départements, communes). Mais il importera de s'intéresser à l'organisation particulière de la Rhénanie-Palatinat, qui n'a pas connu de réforme administrative comme dans les régions voisines, et qui a conservé une division intermédiaire, celle du district. Les Länder sont, en effet, partiellement responsables de la répartition des compétences politiques et administratives entre les différents échelons territoriaux. Il faut noter également que l'attribution des compétences peut être nuancée suivant la taille des communes et leur place dans la hiérarchie urbaine, contrairement à la logique d'égalité républicaine qui prédomine en France.

Illustration 2 - Les communes de moins de cent habitants dans les Länder allemands

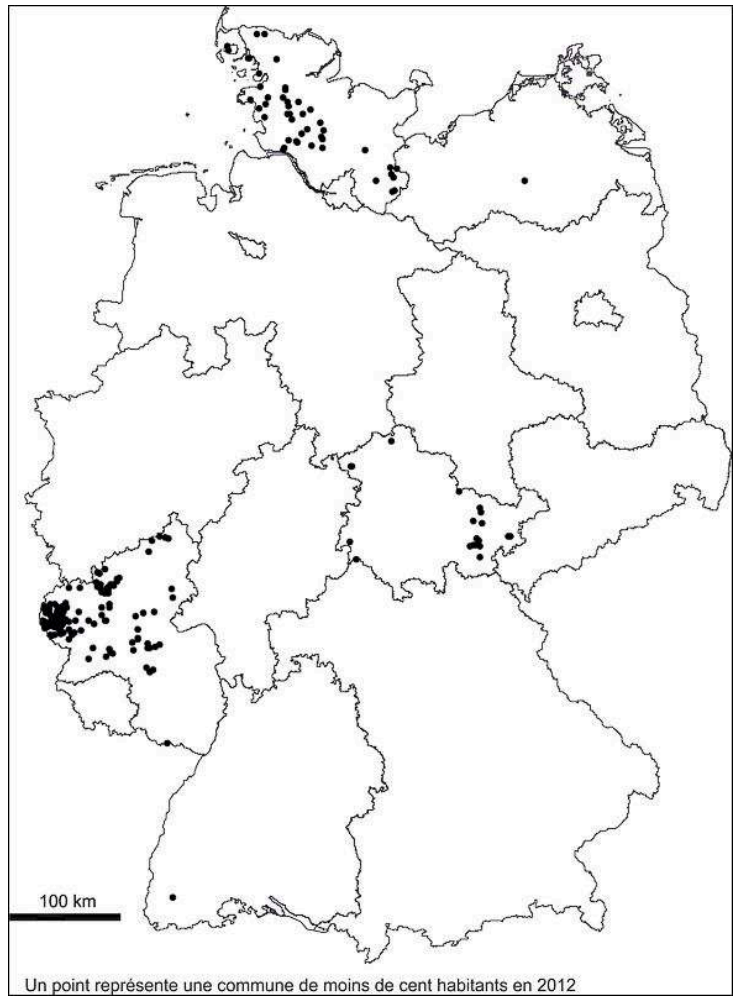

Source : EUROSTAT 2012. 


\section{Le Portugal : un double niveau local bien établi}

40 Ce cas est intéressant dans la mesure où, comme évoqué plus haut, le système administratif reconnaît un niveau infra municipal, celui de la paroisse (freguesia). Correspondant à un maillage fin (4 260 entités au total, soit une moyenne de $21,7 \mathrm{~km}^{2}$ et 2440 habitants, des chiffres légèrement supérieurs à ceux de la France), cette dernière a des compétences limitées: entretien des voies publiques, réalisation des travaux publics, mise en œuvre du recensement électoral et gestion des biens propres. Une série d'autres compétences sont partagées entre les paroisses et les municipalités (305 concelhos, soit approximativement l'équivalent du canton français). Les deux échelons sont gouvernés par des assemblées élues au suffrage universel direct (source : Bruxelles, Comité des Régions). Parmi les freguesias, un petit nombre (115) ont moins de cent habitants, et quelques-unes (21) moins de cinquante au dernier recensement (2011). Ces chiffres étaient respectivement de 62 et 6 en 2001, ce qui traduit un déclin démographique considérable dans les régions concernées. Il s'agit principalement du nord-est du pays (illustration 3). Par ailleurs, une des particularités du système portugais est que ce double niveau local est en place depuis déjà longtemps : le nombre de municipalités n'a pratiquement pas évolué depuis le sortir de la Seconde Guerre mondiale (Conseil de l'Europe, 1995).

Illustration 3 - Les freguesia portugaises de moins de 100 habitants



Source : Eurostat 2012.

41 La répartition des très petites unités administratives locales en Europe montre d'abord que celles-ci ne sont pas l'apanage d'un type particulier de maillage. Elles apparaissent dans des pays appliquant une structure territoriale "éclatée » (dans la majorité des cas), mais aussi là où les moyennes nationales sont relativement élevées, comme en 
Espagne. Seuls les États ayant conduit les réformes les plus radicales n'en présentent qu'à titre exceptionnel (une commune en Belgique). En somme, la présence ou l'absence de très petites entités, et plus généralement la répartition des collectivités par strates de population, introduit une variable supplémentaire dans la diversité des situations nationales.

\section{Une typologie synthétique des maillages locaux européens}

42 Les données élémentaires qualifiant le morcellement administratif local, auxquelles nous incorporons le nombre et la part des communes de moins de cinquante habitants, nous permettent de réaliser une première typologie des maillages locaux européens. Pour cela, nous nous appuyons sur une classification automatique intégrant les six variables suivantes: population moyenne des entités, superficie moyenne, population médiane, écart-type de la population, nombre et part des unités de moins de cent habitants. D'emblée, la partition en sept type est la plus efficiente, au regard des données (maximisation de la variance interclasse, minimisation des variances internes). Le tableau 3 rend compte du résultat obtenu.

Tableau 3 - Typologie par classification automatique des maillages locaux européens

\begin{tabular}{|c|c|c|}
\hline Classe & Pays & Caractéristiques principales \\
\hline 1 & $\begin{array}{l}\text { Bulgarie } \\
\text { France } \\
\text { République } \\
\text { Tchèque }\end{array}$ & \\
\hline 2 & $\begin{array}{l}\text { Grèce } \\
\text { Hongrie } \\
\text { Luxembourg } \\
\text { Islande } \\
\text { Lituanie } \\
\text { Suisse }\end{array}$ & $\begin{array}{l}\text { Trames peu réformées, nombreuses très petites unités, moyennes faibles } \\
\text { de population et de superficie; le tout dans des proportions un peu } \\
\text { moindres pour la classe } 2 \text {. }\end{array}$ \\
\hline 3 & $\begin{array}{l}\text { Chypre } \\
\text { Danemark } \\
\text { Irlande } \\
\text { Liechtenstein } \\
\text { Malte } \\
\text { Portugal } \\
\text { Royaume-Uni } \\
\text { Slovaquie }\end{array}$ & $\begin{array}{l}\text { Trames très morcelées, avec la caractéristique d'écarts-type faibles. Cela } \\
\text { s'explique : pour Chypre, le Liechtenstein, Malte et la Slovaquie, par } \\
\text { l'absence de grande métropole; pour le Danemark, l'Irlande, le Portugal et } \\
\text { le Royaume-Uni, par le fait que les circonscriptions locales découpent auss } \\
\text { les grandes villes, dans la mesure où elles jouent un rôle de circonscription } \\
\text { électorale. }\end{array}$ \\
\hline
\end{tabular}




\begin{tabular}{|l|l|l|}
\hline 4 & $\begin{array}{l}\text { Autriche } \\
\text { Croatie } \\
\text { Italie } \\
\text { Norvège } \\
\text { Roumanie }\end{array}$ & $\begin{array}{l}\text { Écarts-types importants ; pas ou peu de très petites communes ; les autres } \\
\text { variables sont à des niveaux intermédiaires. }\end{array}$ \\
\hline 5 & $\begin{array}{l}\text { Allemagne } \\
\text { Espagne } \\
\text { Finlande } \\
\text { Lettonie } \\
\text { Pologne }\end{array}$ & $\begin{array}{l}\text { Trames caractérisées par des moyennes assez élevées de population et de } \\
\text { superficie ; écarts-types très importants liés à des différenciations } \\
\text { région (ou urbain-rural) significatives. }\end{array}$ \\
\hline 6 & $\begin{array}{l}\text { Belgique } \\
\text { Estonie } \\
\text { Slovénie }\end{array}$ & $\begin{array}{l}\text { Communes relativement peuplées en moyenne ; trames plus homogènes, } \\
\text { les très petites communes sont exceptionnelles. }\end{array}$ \\
\hline 7 & $\begin{array}{l}\text { Pays-Bas } \\
\text { Suède }\end{array}$ & $\begin{array}{l}\text { Communes très peuplées en moyenne (trame fortement réformée en } \\
\text { Suède, partiellement aux Pays-Bas mais dans un contexte de fortes densités }\end{array}$ \\
\hline
\end{tabular}

Cette typologie présente l'intérêt de hiérarchiser assez clairement les pays d'Europe au regard des données globales de leur trame administrative la plus fine. Cependant, nous voyons bien qu'elle n'est pas totalement satisfaisante. Il est important d'y adosser les clés de compréhension des maillages abordées dans la deuxième partie de cet article : le caractère plus ou moins réformé de la trame, les différenciations intra-étatiques, les attributions fonctionnelles des unités administratives locales. Nous en avons tenté une synthèse dans le tableau 4.

Tableau 4 - Une grille de lecture des maillages locaux européens

\begin{tabular}{|l|l|l|l|l|}
\hline \multirow{2}{*}{ Classe } & Pays & $\begin{array}{l}\text { Impact des réformes sur } \\
\text { la trame de base }\end{array}$ & $\begin{array}{l}\text { Différenciation } \\
\text { infranationale }\end{array}$ & $\begin{array}{l}\text { Fonction de la maille } \\
\text { locale }\end{array}$ \\
\hline \multirow{4}{*}{1} & Bulgarie & Faible & Faible & $\begin{array}{l}\text { Electorale } \\
\text { administrative }\end{array}$ \\
\cline { 2 - 5 } & France & Faible & Faible & Collectivité locale \\
\cline { 2 - 6 } & Tchèque & Faible & Faible & Collectivité locale \\
\hline \multirow{2}{*}{2} & Grèce & Faible & Faible & Administrative \\
\cline { 2 - 5 } & Hongrie & Faible & Faible & Collectivité locale \\
\cline { 2 - 5 } & Luxembourg & Faible & Faible & Collectivité locale \\
\cline { 2 - 5 } & Islande & Faible & Moyenne & Collectivité locale \\
\cline { 2 - 5 } & & &
\end{tabular}




\begin{tabular}{|c|c|c|c|c|}
\hline & Lituanie & Faible & Faible & $\begin{array}{l}\text { Electorale / } \\
\text { administrative }\end{array}$ \\
\hline & Suisse & Modéré & Forte & Collectivité locale \\
\hline \multirow{8}{*}{3} & Chypre & Faible & Faible & $\begin{array}{l}\text { Variable selon la } \\
\text { taille }\end{array}$ \\
\hline & Danemark & Faible & Faible & Administrative \\
\hline & Irlande & Faible & Faible & Electorale \\
\hline & Liechtenstein & Faible & Faible & Collectivité locale \\
\hline & Malte & Faible & Faible & Collectivité locale \\
\hline & Portugal & Faible & Forte & $\begin{array}{l}\text { Electorale / } \\
\text { administrative }\end{array}$ \\
\hline & Royaume-Uni & Faible & Faible & Electorale \\
\hline & Slovaquie & Faible & Faible & Collectivité locale \\
\hline & Autriche & Modéré & Forte & Collectivité locale \\
\hline & Croatie & Faible & Faible & Collectivité locale \\
\hline 4 & Italie & Faible & Forte & Collectivité locale \\
\hline & Norvège & Modéré & Faible & Collectivité locale \\
\hline & Roumanie & Fort & Moyenne & Collectivité locale \\
\hline \multirow{5}{*}{5} & Allemagne & Fort & Forte & Collectivité locale \\
\hline & Espagne & Faible & Forte & Collectivité locale \\
\hline & Finlande & Fort & Moyenne & Collectivité locale \\
\hline & Lettonie & Fort & Faible & Collectivité locale \\
\hline & Pologne & Fort & Faible & Collectivité locale \\
\hline \multirow{3}{*}{6} & Belgique & Fort & Faible & Collectivité locale \\
\hline & Estonie & Fort & Moyenne & Collectivité locale \\
\hline & Slovénie & Fort & Faible & Collectivité locale \\
\hline \multirow{2}{*}{7} & Pays-Bas & Modéré & Faible & Collectivité locale \\
\hline & Suède & Fort & Moyenne & Collectivité locale \\
\hline
\end{tabular}


44

Les éléments apportés par ce tableau soulignent bien la progression des trames les plus éclatées vers les maillages les plus lâches. Ils tendent à confirmer la pertinence de la classification automatique, tout en y apportant des nuances indispensables. Les trois premières catégories peuvent aisément être partagées en deux grands types.

D'une part, des États conservent un premier niveau de collectivités territoriales très morcelé, dont la France fait évidemment partie. On y retrouve aussi plusieurs pays d'Europe centrale et orientale (République Tchèque, Hongrie, Slovaquie), comptant souvent un certain nombre de très petites communes, et des petits pays aux trames assez homogènes (Luxembourg, Malte, Liechtenstein, Islande). La Suisse être apparentée à cette classe, même si quelques cantons ont déjà réformé leur trame locale. D'autre part, une série de pays n'accordent pas le statut de collectivité locale au premier niveau de leur organisation territoriale, le cantonnant à certaines fonctions, généralement électorales (élection de représentants au conseil de la collectivité de niveau supérieur), souvent administratives (notamment pour la gestion de l'état civil). Une différence importante entre de tels maillages et les trames du type précédent est le fait que le plus souvent, les villes sont elles-mêmes subdivisées en de multiples circonscriptions de quartier ou d'arrondissement. On notera enfin qu'à Chypre, les unités administratives locales sont réparties entre des municipalités, qui ont le statut de collectivité locale, et des communautés qui ne l'ont pas.

Les quatre dernières catégories de la classification automatiques peuvent à nouveau être partagées en deux grands types. D'une part, quelques pays présentent une trame intermédiaire, ayant peu ou modérément évolué (la réduction du nombre de municipalités depuis 1950 ne dépasse pas 50 \%). Il s'agit de l'Autriche, la Croatie, l'Italie, la Norvège, l'Espagne. On peut y adjoindre les Pays-Bas qui, malgré des regroupements plus fréquents, conservent des mailles relativement proches de la trame urbaine. D'autre part, quelques pays ont profondément remanié leurs trames locales au cours des dernières décennies, en supprimant presque tout statut aux entités d'origine. Il s'agit bien sûr de la Suède, de la Belgique, de l'Allemagne (malgré le maintien de la trame initiale dans certains Länder), de la Roumanie, de la Pologne, la Slovénie, la Lettonie, l'Estonie, la Finlande.

Notre typologie finale peut donc être résumée dans le tableau suivant :

Tableau 5 - Typologie synthétique des maillages administratifs locaux européens

\begin{tabular}{|l|l|l|}
\hline Type & Caractéristiques & Pays \\
\hline I & $\begin{array}{l}\text { Trame morcelée, peu réformée, calquée sur la } \\
\text { répartition de l'habitat; unités locales souvent peu } \\
\text { peuplées }\end{array}$ & $\begin{array}{l}\text { France, République Tchèque, Hongrie, } \\
\text { Luxembourg, Islande, Suisse, Slovaquie, } \\
\text { Liechtenstein, Malte }\end{array}$ \\
\hline II & $\begin{array}{l}\text { Trame morcelée, mais les unités locales n'ont pas } \\
\text { le statut de collectivité territoriale } \\
\text { (circonscriptions) }\end{array}$ & $\begin{array}{l}\text { Bulgarie, Grèce, Lituanie, Danemark, } \\
\text { Irlande, Portugal, Royaume-Uni, } \\
\text { Chypre }\end{array}$ \\
\hline III & $\begin{array}{l}\text { Trame peu ou moyennement réformée, } \\
\text { collectivités locales traditionnellement peuplées ; } \\
\text { parfois de fortes disparités internes }\end{array}$ & $\begin{array}{l}\text { Autriche, Croatie, Italie, Norvège, } \\
\text { Espagne, Pays-Bas }\end{array}$ \\
\hline
\end{tabular}


\begin{tabular}{|l|l|l|}
\hline IV & Trame réformée par fusion large des municipalités & $\begin{array}{l}\text { Roumanie, Allemagne, Finlande, } \\
\text { Lettonie, Pologne, Belgique, Estonie, } \\
\text { Slovénie, Suède }\end{array}$ \\
\hline
\end{tabular}

49 Établir une telle typologie n'est pas une chose facile, et certains choix mériteraient certainement d'être discutés. Il semble que son principal apport par rapport aux tableaux habituellement présentés est de choisir de comparer des mailles équivalentes (niveau LAU 2 d'Eurostat), et non des statuts équivalents. Une des limites d'une telle approche réside dans la définition des unités administratives prises en compte par l'office statistique européen. En effet, plusieurs pays ont un niveau d'entités subdivisant encore le LAU 2. Le cas le plus significatif est sans doute celui des civil parishes (" paroisses civiles») qui couvrent l'essentiel de l'Angleterre rurale, et seraient peut-être la circonscription d'outre-manche la plus proche de la commune française, avec ses élections locales, son conseil de paroisse élu pour la gestion des affaires courantes (Wollmann, 2008). En France, les quelques centaines de communes associées créées dans les années 1970 suite à la loi Marcellin de 1971, et les centaines de communes déléguées qui voient le jour dans les années 2010 suite aux lois sur la mise en place des communes nouvelles, ne font encore l'objet que d'une prise en compte très limitée dans l'étude des systèmes administratifs territoriaux. Or, si ces dernières étaient appelées à se généraliser dans les années à venir, notre maillage territorial ressemblerait de plus en plus à ceux du deuxième type.

50 Une autre limite à cette typologie, liée à notre approche de géographe, est de ne considérer que de façon secondaire l'évolution des statuts des collectivités locales. Or, il est évident que la forme du maillage n'est pas la seule clé de son efficacité ou de sa pertinence. Dans certains pays, des réformes importantes touchant aux compétences des collectivités ont pu être observées. En France, les transferts de compétences des communes vers les intercommunalités ont changé, en grande partie, le périmètre des actions strictement municipales, malgré une trame locale figée en apparence.

\section{Conclusion}

51 Ces quelques observations conduisent à s'interroger quant à la spécificité du cas français en matière d'émiettement communal. En effet, si des régions de forte concentration de très petites communes existent chez nos voisins, les modèles spatiaux liés à ces entités sont-ils pour autant les mêmes ? Posent-ils les mêmes questions quant aux dynamiques locales, et pour la gestion du territoire? À ce niveau, les différences d'organisation administrative entre les États, en particulier les inégales réformes, voire « révolutions » territoriales dont ils ont fait l'objet ont elles permis de régler certains problèmes de développement local plus efficacement dans certains pays?

52 Les quelques cas étudiés plus haut donnent plutôt l'impression que chaque pays présente des particularités notoires, et qu'il est difficile de trouver un véritable dénominateur commun permettant de faire passer la France pour une exception par rapport à une norme qui en fait n'existe pas réellement. Le système hexagonal est sans aucun doute unique, mais sans s'opposer pour autant à un modèle uniforme qui prédominerait ailleurs. En revanche, ce fait n'empêche pas de chercher ailleurs des modèles territoriaux susceptibles d'être plus efficaces et plus dynamiques. Si les 
pouvoirs publics, en France, invoquent volontiers le «modèle allemand », leurs visions du découpage idéal se rapprochent plus souvent des regroupements massifs et uniformes de la Belgique ou de la Suède, tandis que la réalité des campagnes de réformes tendrait davantage vers ce que l'on observe en Suisse, voire au Portugal si les communes nouvelles se généralisaient...

53 Enfin, la compréhension des divergences de trames européennes présente une portée méthodologique : les comparaisons internationales à l'échelle locale, mises en œuvre dans de nombreux travaux d'analyse spatiale, se heurtent à ces biais de structure, qui se résument par l'écart paradoxal entre, schématiquement, l'Europe des 100000 communes, toujours en mouvement mais qui constitue la référence officielle, et celle, beaucoup plus stable mais méconnue, des 200000 villages.

\section{BIBLIOGRAPHIE}

Aubelle V., 2011. Intercommunalités : des projets aux pratiques. Paris, Berger-Levrault, 618 p.

Bouillot M., Lenormand P., 2002. Villes et campagnes à l'épreuve de l'intercommunalité. Les Carnets de l'observatoire sur les rapports entre le rural et l'urbain, Nanterre, LADYSS, $n^{\circ} 1$, p. 33-60.

Boulineau E., 2003. Maillages administratifs et gestion du territoire en Bulgarie : une lecture géographique. Thèse, Paris,Université Panthéon-Sorbonne, $462 \mathrm{p}$.

Conseil de l'Europe, 1995. La Taille des communes, l'efficacité et la participation des citoyens. Rapports préparés dans le cadre du Comité Directeur des autorités Locales et Régionales (CDLR), Strasbourg, les éditions du Conseil de l'Europe (Communes et régions d'Europe, $\left.\mathrm{n}^{\circ} 56\right), 220 \mathrm{p}$.

Delamarre A., 1989. La Carte des 36000 communes. Mappemonde, Montpellier, Reclus $n^{\circ} 4$, p. 12-16.

Depraz S., 2006. Du territoire identitaire au maillage fonctionnel : la réforme graduelle des communes dans les nouveaux Länder allemands. In A. Bleton-Ruget, N. Commerçon et P. Gonod (dir.), Territoires institutionnels, territoires fonctionnels. Mâcon, Institut de recherche du val de Saône mâconnais, p. 227-235.

Depraz S., 2005. Recompositions territoriales, développement rural et protection de la nature dans les campagnes d'Europe centrale post-socialiste. Thèse, Montpellier, Université Paul-Valéry, $526 \mathrm{p}$.

Gabriel-Oyhamburu K, 2000. La Recomposition territoriale de l'Espagne et la mémoire de ses territoires. Revue de géographie alpine, Grenoble, Institut de Géographie Alpine, tome $88, \mathrm{n}^{\circ} 1$, p. 17-34.

Giraut F., 2002. L'exception territoriale française sous influence européenne ? Resituer la dynamique du système français d'administration territoriale. L'Information géographique, Paris, SEDES, vol. 66, n² 2, p. 133-161.

Grison J.-B., 2012. Les très petites communes en France : héritage sans avenir ou modèle original?

Clermont-Ferrand, Presses Universitaires Blaise-Pascal, 360 p. 
Guigou J.-L., 2000. La Mutation silencieuse des territoires / différents articles, interviews, interventions, notes de J.-L. Guigou, de 1990 à 1999. Paris, DATAR, ministère de l'aménagement du territoire et de l'environnement (2 tomes, non paginé).

Martin M.-R., 1995. Size of municipalities, efficiency, and citizen participation: a cross-European perspective. Environment and Planning C: Government and Policy, London, Pion, vol. 13, $\mathrm{n}^{\circ} 4$, p. 441-458.

Maurel M-C., 2005. Les collectivités locales d'Europe centre-orientale : des territoires politiques en recomposition. In Rey V., Coudroy De Lille L. et Boulineau E. (dir.), L'élargissement de l'Union européenne : réformes territoriales en Europe centrale et orientale. Paris : L'Harmattan, p. 81-94.

Maurel M.-C., 1984. Pour une géopolitique du territoire. L'étude du maillage politicoadministratif. Hérodote, Paris, La Découverte, n³3-34, p. 131-143.

Meynier A., 1945. La commune rurale française. Annales de géographie, Paris, Armand Colin, $\mathrm{n}^{\circ} 295$, p. 161-179.

Nates Cruz B., 2008. Productions territoriales et innovation des traditions : des "natifs" face à l'implantation des Européens du nord - étude comparée en zones rurales française et espagnole. Les étrangers dans les campagnes / Actes du colloque franco-britannique de géographie rurale, Clermont-Ferrand, Presses Universitaires Blaise-Pascal, coll. CERAMAC n 25, p. 563-579.

Reitel F., 1973. Un massif central rhénan et ses bordures. Tthèse, Strasbourg, faculté des Lettres et Sciences Humaines, 2 tomes, $716 \mathrm{p}$.

Sanz Gomez M., 2005. Les Collectivités territoriales en Espagne : entre continuité et rénovation. Revue d'économie régionale et urbaine, Poitiers, IERF, $\mathrm{n}^{\circ}$ 1, p. 31-56.

Sivignon M. et al., 2003. Atlas de la Grèce. Paris, La Documentation Française, Montpellier, UMR ESPACE-GDR Libergéo, coll. Dynamiques du territoire, 190 p..

Wollmann H., 2008. Comparing Local Government Reforms in England, Sweden, France and Germany. Ludwigsburg, Fondation Wüstenrot, 498 p., rapport consultable sur le site http:// www.wuestenrot-stiftung.de

Ziller J., 2000. Fragmentation / Participation : quelle bonne dimension ? Pouvoirs : Revue française d'études constitutionnelles et politiques, Paris, Seuil, nº 95, p. 19-31.

\section{NOTES}

1. Afin de ne pas introduire de biais dans l'analyse, nous n'incluons dans cette comparaison que les communes de Métropole.

2. Chiffres du Conseil de l'Europe (1995).

3. Source : Statistisches Landesamt Rheinland-Pfalz, 2004 - population au 31 décembre 2003. 


\section{RÉSUMÉS}

Cet article questionne la singularité du maillage communal français par rapport aux autres découpages locaux en Europe. À partir de la comparaison des données nationales, mais aussi de la morphologie des maillages, nous avons proposé une première approche de leur diversité. Dans un deuxième temps, l'approche par les très petites communes fournit un complément d'analyse intéressant, qui tend à renforcer la diversité des configurations. Les études de cas abordées font état, en outre, de variations infranationales fréquentes. Ces analyses débouchent sur un essai de typologie renouvelée des maillages européens, au sein de laquelle le caractère exceptionnel de la trame française peut être en partie relativisé, même si cette trame conserve, à l'instar d'autres, des singularités propres. La France est loin d'être le seul pays d'Europe à compter un maillage morcelé et des entités très peu peuplées. Et quel que soit le système administratif pratiqué, se pose la question de la prise en compte des plus petites communautés locales.

This paper questions the particularities of French local governance in comparison with other local administrative network in Europe. National data are first considered. The national administrative profiles shows different categories of countries, and some specificities in the French case. Second, a distinctive approach to the smaller municipalities in each country allows a different vision of the divergences. Moreover, a look at some national case studies, sometimes shows important regional variations. Third, all these observations result in a new typology of European local administrative systems. With this typology, the French exception becomes more nuanced, in regards to several other exceptions. France is not the only country to include very small administrative divisions. Furthermore, all local administrative systems have to take the smaller local communities into consideration.

INDEX

Keywords : local administrative units, municipal framework, administrative geography, territorial governance, France, Europe

Mots-clés : unité administrative locale, maillage communal, géographie administrative, gouvernance territoriale, France, Europe

Thèmes : Sur le Champ - Sur le Terrain

\section{AUTEUR}

\section{JEAN-BAPTISTE GRISON}

Jean-Baptiste Grison, jbgrison1@gmail.com, est Docteur en géographie, CERAMAC, ClermontUniversité. 